\title{
PROCYCLIC COVERINGS OF COMMUTATORS IN PROFINITE GROUPS
}

\author{
GUSTAVO A. FERNÁNDEZ-ALCOBER, MARTA MORIGI, \\ AND PAVEL SHUMYATSKY
}

\begin{abstract}
We consider profinite groups in which all commutators are contained in a union of finitely many procyclic subgroups. It is shown that if $G$ is a profinite group in which all commutators are covered by $m$ procyclic subgroups, then $G$ possesses a finite characteristic subgroup $M$ contained in $G^{\prime}$ such that the order of $M$ is $m$-bounded and $G^{\prime} / M$ is procyclic. If $G$ is a pro- $p$ group such that all commutators in $G$ are covered by $m$ procyclic subgroups, then $G^{\prime}$ is either finite of $m$-bounded order or procyclic.
\end{abstract}

\section{INTRODUCTION}

A covering of a group $G$ is a family $\left\{S_{i}\right\}_{i \in I}$ of subsets of $G$ such that $G=\bigcup_{i \in I} S_{i}$. If $\left\{H_{i}\right\}_{i \in I}$ is a covering of $G$ by subgroups, it is natural to ask what information about $G$ can be deduced from properties of the subgroups $H_{i}$. In the case where the covering is finite actually quite a lot about the structure of $G$ can be said. In particular, as was first pointed out by Baer (see [11, p. 105]), a group covered by finitely many cyclic subgroups is either cyclic or finite. FernándezAlcober and Shumyatsky proved that if $G$ is a group in which the set of all commutators is covered by finitely many cyclic subgroups, then the derived group $G^{\prime}$ is either finite or cyclic [5]. Later, in [3], Cutolo and Nicotera showed that if $G$ is a group in which the set of all $\gamma_{j^{-}}$ commutators is covered by finitely many cyclic subgroups, then $\gamma_{j}(G)$ is finite-by-cyclic. They also showed that $\gamma_{j}(G)$ can be infinite and not cyclic. It is still unknown whether a similar result holds for the derived words $\delta_{j}$.

2010 Mathematics Subject Classification. 20E18; $20 \mathrm{~F} 14$.

Key words and phrases. Profinite groups; procyclic subgroups; commutators.

The first and second authors are supported by the Spanish Government, grant MTM2011-28229-C02-02. The first and third authors are supported by the Brazilian and Spanish Governments, under the project with the following references: Capes/DGU 304/13; PHB2012-0217-PC. The first author is also supported by the Basque Government, grant IT753-13. The second author is also supported by INDAM (GNSAGA). 
Recall that a profinite group is a topological group that is isomorphic to an inverse limit of finite groups. The textbooks [10] and [12] provide a good introduction to the theory of profinite groups. In the context of profinite groups all the usual concepts of group theory are interpreted topologically. In particular, the derived group $G^{\prime}$ of a profinite group $G$ is the closed subgroup generated by all commutators in $G$.

In this paper we examine profinite groups in which all commutators are covered by finitely many procyclic subgroups. Our natural expectation was that the derived subgroup in such a group should either be finite or procyclic, but this turned out to be false. Indeed, let $A$ be a finite group such that $A^{\prime}$ is noncyclic of order four, and let $B$ be a pro- $p$ group such that $B^{\prime}$ is infinite procyclic, where $p$ is an odd prime. Then it is easy to see that $G=A \times B$ is a profinite group in which $G^{\prime}$ is infinite, not procyclic, and can be covered by 3 procyclic subgroups.

However, we can prove the following result.

Theorem A. Let $m$ be a positive integer and let $G$ a profinite group in which all commutators are covered by $m$ procyclic subgroups. Then $G$ possesses a finite characteristic subgroup $M$ contained in $G^{\prime}$ such that the order of $M$ is $m$-bounded and $G^{\prime} / M$ is procyclic.

As usual, we use the expression " $a$-bounded" to mean "bounded from above by some function depending only on the parameter $a$ ".

Further, we concentrate on pro- $p$ groups in which all commutators are covered by finitely many procyclic subgroups. In this case our initial expectation that $G^{\prime}$ is either finite or procyclic has been confirmed.

Theorem B. Let $p$ be a prime and let $G$ be a pro- $p$ group such that all commutators in $G$ are covered by $m$ procyclic subgroups. Then $G^{\prime}$ is either finite of $m$-bounded order or procyclic.

The above results are not the first that deal with coverings of wordvalues in profinite groups. For a family of group words $w$ it was shown in [1] that if $G$ is a profinite group in which all $w$-values are contained in a union of finitely many closed subgroups with a prescribed property, then the verbal subgroup $w(G)$ has the same property as well. More recently the results obtained in [1] have been extended to profinite groups in which all $w$-values are contained in a union of countably many closed subgroups [4]. Quite possibly, for profinite groups in which the commutators are covered by countably many procyclic subgroups some analogues of Theorem A and Theorem B hold true.

Though profinite groups constitute the main topic of the present study, the above results also have a bearing on the case of abstract groups. As we have already mentioned, the main result in [5] says that 
if $G$ is an abstract group whose commutators are covered by finitely many cyclic subgroups, then $G^{\prime}$ is either finite or cyclic. Now we can deduce the following additional information.

Theorem C. Let $G$ be a group that possesses $m$ cyclic subgroups whose union contains all commutators of $G$. Then $G$ has a characteristic subgroup $M$ contained in $G^{\prime}$ such that the order of $M$ is $m$-bounded and $G^{\prime} / M$ is cyclic.

Of course, the information provided by Theorem $\mathrm{C}$ is meaningful only in the case where $G^{\prime}$ is finite, since otherwise $G^{\prime}$ is cyclic.

\section{Finite Groups With COMMUtATORS COVERED By FEW CYCLIC SUBGROUPS}

We start with some elementary lemmas.

Lemma 2.1. Let $n \geq 1$ be a positive integer and let $H$ be a characteristic finite nilpotent subgroup of a group $G$. Assume that for every prime $p$ dividing the order of $H$ the Sylow $p$-subgroup $P$ of $H$ has a characteristic subgroup $M_{p}$, of order at most $n$, such that $P / M_{p}$ is cyclic. Then $G$ possesses a characteristic subgroup $M$ contained in $H$ such that the order of $M$ is $n$-bounded and $H / M$ is cyclic.

Proof. We take $M$ to be the product of all $M_{p}$, where $p$ ranges through the set of all prime divisors of the order of $H$. Obviously, $M_{p}=1$ whenever $p \geq n+1$ and therefore the order of $M$ is less than $n^{n}$. It is clear that, being the product of characteristic subgroups, $M$ is characteristic. The quotient $H / M$ is a nilpotent group with cyclic Sylow subgroups and therefore $H / M$ is cyclic. The proof is complete.

Lemma 2.2. Let $H$ be a characteristic subgroup of an abstract (resp. profinite) group $G$. Suppose that $H$ possesses a normal finite subgroup $N$ such that $H / N$ is cyclic (resp. procyclic). Then $G$ has a characteristic subgroup $M$ contained in $H$ such that the order of $M$ is at most $|N|^{2}$ and $H / M$ is cyclic (resp. procyclic).

Proof. Take $M$ to be the subgroup generated by all elements of $H$ of order dividing $|N|$. It is clear that $M$ is a characteristic subgroup in $G$ containing $N$. Since the quotient $M / N$ is cyclic (resp. procyclic) and generated by elements of order dividing $|N|$, it has order dividing $|N|$. It follows that the order of $M$ is at most $|N|^{2}$, as required.

The following lemma is taken from [2]. It will play an important role in our arguments. 
Lemma 2.3. Let $G$ be a finite noncyclic p-group that can be covered by $m$ cyclic subgroups. Then the order of $G$ is $m$-bounded.

Recall that in a group $G$ the subgroup $\gamma_{\infty}(G)$ is the intersection of all $\gamma_{i}(G)$ for $i \in \mathbb{N}$. Clearly, a finite group $G$ is nilpotent if and only if $\gamma_{\infty}(G)=1$. It is an easy exercise to show that if $G$ is a finite group, then $\gamma_{\infty}(G)$ is generated by the commutators $[x, y]$ such that $x, y$ are elements of $G$ having mutually coprime orders. The following theorem was proved in [2].

Theorem 2.4. Let $G$ be a finite group that possesses $m$ cyclic subgroups whose union contains all commutators $[x, y]$ such that $x, y$ are elements of $G$ having mutually coprime orders. Then $\gamma_{\infty}(G)$ has a subgroup $\Delta$ such that

(i) $\Delta$ is normal in $G$;

(ii) $|\Delta|$ is m-bounded;

(iii) $\gamma_{\infty}(G) / \Delta$ is cyclic.

Further, we will require the following special case of a result of Guralnick [6, Theorem A].

Theorem 2.5. Let $G$ be a finite group in which $G^{\prime}$ is an abelian pgroup generated by at most two elements. Then every element of $G^{\prime}$ is a commutator.

We will now start our analysis of finite groups in which commutators are covered by at most $m$ cyclic subgroups. We recall that a finite group $G$ has rank $r$ if $r$ is the least integer such that every subgroup of $G$ can be generated by at most $r$ elements.

Lemma 2.6. Let $G$ be a finite nilpotent group of class 2 that possesses $m$ cyclic subgroups whose union contains all commutators of $G$. Then $G^{\prime}$ has a characteristic subgroup $M$ such that $|M|$ is m-bounded and $G^{\prime} / M$ is cyclic.

Proof. By Lemma 2.1 it is sufficient to show that the claim is correct for each Sylow subgroup of $G$. Therefore we can assume that $G$ is a $p$-group for some prime $p$. Since $G$ is of class 2 , it follows that for each element $y \in G$ the subgroup $[G, y]$ consists entirely of commutators. By Lemma 2.3 there exists a bound $\beta$ such that either $[G, y]$ is cyclic or $|[G, y]| \leq \beta$. Let $M$ be the product of all subgroups of $G^{\prime}$ whose order is at most $\beta$. Since $G^{\prime}$ is an abelian group with at most $m$ generators, the rank of $G^{\prime}$ is at most $m$. It follows that the order of $M$ is bounded as well. We pass to the quotient $G / M$ and we obtain that $[G, y]$ is cyclic for all $y \in G$. Suppose that $G^{\prime}$ is not cyclic. Passing to $G / \Phi\left(G^{\prime}\right)$, 
we assume that $G^{\prime}$ is elementary abelian. We can choose $x, y \in G$ such that $[G, x]$ and $[G, y]$ are both nontrivial and $[G, x] \neq[G, y]$. Now choose any element $t \in G$ which does not belong to $C_{G}(x) \cup C_{G}(y)$. Such an element $t$ exists because a group cannot be the union of two proper subgroups. Then $[G, t]=[G, x]$ because these are both cyclic groups of order $p$ containing $[x, t] \neq 1$. Similarly $[G, t]=[G, y]$, a contradiction.

In what follows we write $O_{p^{\prime}}(X)$ to denote the largest normal $p^{\prime}$ subgroup of a finite group $X$.

Lemma 2.7. Let $G$ be a finite metabelian group that possesses $m$ cyclic subgroups whose union contains all commutators of $G$. Then $G^{\prime}$ has a characteristic subgroup $M$ such that $|M|$ is $m$-bounded and $G^{\prime} / M$ is cyclic.

Proof. By Lemma 2.1 it is sufficient to show that each Sylow subgroup of $G^{\prime}$ possesses a characteristic subgroup with the required properties. Let $p$ be a prime divisor of the order of $G^{\prime}$. Passing to the quotient $G / O_{p^{\prime}}\left(G^{\prime}\right)$ we can assume that $G^{\prime}$ is a $p$-group. Let $a \in G^{\prime}$ and $b \in G$. It is clear that each element of $\langle[a, b]\rangle$ has form $\left[a^{i}, b\right]$. If $x \in G$ we have $\left[x a^{i}, b\right]=[x, b]^{a^{i}}\left[a^{i}, b\right]=[x, b]\left[a^{i}, b\right]$ and so every element in the coset $[x, b]\langle[a, b]\rangle$ is a commutator. Thus, the coset $[x, b]\langle[a, b]\rangle$ is covered by $m$ cyclic subgroups. It follows that for some $1 \leq i \neq j \leq m+1$ one of the elements $[x, b]\left[a^{i}, b\right]$ and $[x, b]\left[a^{j}, b\right]$ is a power of the other. For simplicity, assume that $C_{1}=\left\langle[x, b]\left[a^{i}, b\right]\right\rangle$ contains $[x, b]\left[a^{j}, b\right]$. Then also $\left[a^{j-i}, b\right] \in C_{1}$. Therefore the subgroup $\left\langle C_{1},[a, b]\right\rangle$ decomposes as a direct product $C_{2} \times D_{2}$, where $C_{2}$ is cyclic and $D_{2}$ is cyclic of order at most $m$. Let $D$ be the product of all subgroups of $G^{\prime}$ whose order is at most $m$. Since $G^{\prime}$ is an abelian group with at most $m$ generators, the rank of $G^{\prime}$ is at most $m$. It follows that the order of $D$ is at most $m^{m}$. We pass to the quotient $G / D$ and we see that

$$
\langle[a, b],[x, b]\rangle \text { is cyclic for all } a \in G^{\prime} \text { and } b, x \in G \text {. }
$$

Note that (2.1) implies in particular that $\left[G^{\prime}, y\right]$ is cyclic for all $y \in G$.

Let us now show that $\gamma_{3}(G)$ is cyclic. We can pass to $G / \Phi\left(\gamma_{3}(G)\right)$ and assume that $\gamma_{3}(G)$ is elementary abelian. Choose $y \in G$ such that $\left[G^{\prime}, y\right] \neq 1$ and $x$ outside $C_{G}(y)$. Since $\left[G^{\prime}, y\right]$ is of order $p$, by (2.1) we have $\left[G^{\prime}, y\right] \leq\langle[x, y]\rangle$. The same argument shows that $\left[G^{\prime}, x\right] \leq$ $\langle[x, y]\rangle$. Using that $\left[G^{\prime}, x\right]$ is of order at most $p$, we conclude that $\left[G^{\prime}, x\right] \leq\left[G^{\prime}, y\right]$. This happens for all $x$ outside $C_{G}(y)$. Since the set of all such $x$ outside $C_{G}(y)$ generates the whole group $G$, it follows that $\gamma_{3}(G)=\left[G^{\prime}, y\right]$. 
Thus, indeed $\gamma_{3}(G)$ is cyclic (we no longer assume that $\gamma_{3}(G)$ is elementary abelian). By Lemma 2.6, $G^{\prime} / \gamma_{3}(G)$ has a characteristic subgroup $K / \gamma_{3}(G)$ such that $\left|K / \gamma_{3}(G)\right|$ is $m$-bounded and $G^{\prime} / K$ is cyclic. We see that $K$ is an abelian subgroup containing a cyclic subgroup of bounded index. The subgroup of $K$ generated by all elements of order at most the exponent of $K / \gamma_{3}(G)$ is characteristic and has bounded order. By factoring this subgroup we may assume that $K / \gamma_{3}(G)$ is cyclic and so $G^{\prime} / \gamma_{3}(G)$ has rank at most two. Now Theorem 2.5 tells us that every element of $G^{\prime} / \gamma_{3}(G)$ is a commutator. Therefore Lemma 2.3 shows that $G^{\prime} / \gamma_{3}(G)$ either is cyclic or has $m$-bounded order.

Suppose that $G^{\prime} / \gamma_{3}(G)$ is cyclic. Taking into account that $\gamma_{3}(G)$ is cyclic as well, we conclude that $G^{\prime}$ is 2-generator. By Theorem 2.5 every element of $G^{\prime}$ is a commutator. Therefore Lemma 2.3 shows that $G^{\prime}$ either is cyclic or has $m$-bounded order, whence the lemma follows.

Suppose now that $G^{\prime} / \gamma_{3}(G)$ has $m$-bounded order. We argue as above. Let $X$ be the product of all subgroups of $G^{\prime}$ of order at most $\left|G^{\prime} / \gamma_{3}(G)\right|$. As $G^{\prime}$ has rank at most 3, the subgroup $X$ has bounded order and $G^{\prime} / X$ is cyclic. The proof is complete.

Theorem 2.8. Let $G$ be a finite group that possesses $m$ cyclic subgroups whose union contains all commutators of $G$. Then $G^{\prime}$ has a characteristic subgroup $M$ such that the order of $M$ is $m$-bounded and $G^{\prime} / M$ is cyclic.

Proof. Let $\Delta$ have the same meaning as in Theorem 2.4. By Lemma 2.2. we may assume that $\Delta$ is characteristic in $G$. We can pass to the quotient $G / \Delta$ and suppose that $G$ is soluble with $\gamma_{\infty}(G)$ cyclic. The group $G$ acts on $\gamma_{\infty}(G)$ by conjugation and as the automorphism group of a cyclic group is abelian, it follows that $G^{\prime}$ centralizes $\gamma_{\infty}(G)$. Therefore $G^{\prime}$ is nilpotent. By Lemma 2.1 it is sufficient to show that each Sylow subgroup of $G^{\prime}$ possesses a characteristic subgroup with the required properties. Let $p$ be a prime divisor of the order of $G^{\prime}$. Passing to the quotient $G / O_{p^{\prime}}\left(G^{\prime}\right)$ we can assume that $G^{\prime}$ is a $p$-group.

Next we remark that since $G^{\prime}$ is an $m$-generator $p$-group, the Burnside Basis Theorem [9, III.3.15] shows that $G^{\prime}$ is generated by $m$ commutators. Therefore we can choose at most $2 m$ elements in $G$ such that $G^{\prime}$ is generated by commutators in the chosen elements. Without loss of generality we can assume that $G$ is generated by the chosen elements.

Let $x$ be a commutator. Then any conjugate of $x$ is again a commutator and so it belongs to at least one of the $m$ cyclic subgroups covering the commutators of $G$. Since any finite cyclic subgroup has at most one subgroup of any given order, it follows that the subgroup $\langle x\rangle$ 
has at most $m$ conjugates. Thus, $N_{G}(\langle x\rangle)$ has index at most $m$. Set $T=\cap N_{G}(\langle x\rangle)$, where $x$ ranges over all commutators in $G$. Since $G$ can be generated by $2 m$ elements, it has only boundedly many subgroups of any given index [8, Theorem 7.2.9] and so $T$ has $m$-bounded index in $G$. Also, $T^{\prime} \leq C_{G}(x)$ for every commutator $x$, and so $T^{\prime}$ centralizes $G^{\prime}$. Therefore $T$ is metabelian and the derived length of $G$ is bounded.

We will now use induction on the derived length of $G$. By induction, we can assume that $G^{\prime \prime}$ has a characteristic subgroup $M_{1}$ such that $\left|M_{1}\right|$ is $m$-bounded and $G^{\prime \prime} / M_{1}$ is cyclic. Passing to the quotient over $M_{1}$, we assume that $G^{\prime \prime}$ is cyclic. The group $G$ induces by conjugation an abelian group of automorphisms of $G^{\prime \prime}$. Hence, $G^{\prime}$ centralizes $G^{\prime \prime}$ and thus the nilpotency class of $G^{\prime}$ is at most 2 .

By Lemma 2.7 (applied to $G / G^{\prime \prime}$ ) the derived group $G^{\prime}$ has a characteristic subgroup $M$ containing $G^{\prime \prime}$, and such that $M / G^{\prime \prime}$ has $m$ bounded order while $G^{\prime} / M$ is cyclic. As $|M: Z(M)| \leq\left|M: G^{\prime \prime}\right|$, by the Schur Theorem [11, Theorem 4.12] $M^{\prime}$ has $m$-bounded order as well. Factoring $M^{\prime}$ out we can assume that $M$ is abelian. We can write $M=R \times M_{2}$, where $R$ is a cyclic group and $M_{2}$ is a subgroup of $m$-bounded order. It follows from Lemma 2.2 that $M_{2}$ is contained in a characteristic subgroup of $G$ of $m$-bounded order. Factoring it out, we can assume that $M$ is cyclic. Moreover, $G$ acts on $M$ by conjugation so $\left[G^{\prime}, M\right]=1$. It follows that $G^{\prime} / Z\left(G^{\prime}\right)$ is cyclic. We conclude that $G^{\prime}$ is abelian and the theorem follows from Lemma 2.7.

It is easy to see that under the hypothesis of Theorem 2.8 the order of $G^{\prime}$ cannot be bounded even if we know that $G^{\prime}$ is noncyclic. Indeed, let $A$ be a finite group such that $A^{\prime}$ is noncyclic of order four and let $B$ be a finite group such that $B^{\prime}$ has odd prime order $p$. Set $G=A \times B$. Then $G^{\prime}$ is noncyclic and covered by 3 cyclic subgroups. The order of $G^{\prime}$ is $4 p$ and this tends to infinity when $p$ does so.

However, our next result shows that if $G$ is a $p$-group satisfying the hypothesis of Theorem 2.8 and having noncyclic derived group $G^{\prime}$, then the order of $G^{\prime}$ is $m$-bounded.

Theorem 2.9. Let $p$ be a prime and let $G$ be a finite p-group in which all commutators can be covered by $m$ cyclic subgroups. Then either $G^{\prime}$ is cyclic or the order of $G^{\prime}$ is m-bounded.

Proof. Let us assume that $G^{\prime}$ is not cyclic. By Theorem 2.8 the derived group $G^{\prime}$ contains a characteristic subgroup $M$ of $m$-bounded order such that $G^{\prime} / M$ is cyclic.

We choose in $G$ a normal subgroup $N$ of minimum possible order subject to the condition that $(G / N)^{\prime}$ is cyclic. Then $1 \neq N \subseteq G^{\prime}$, and 
the order of $N$ is $m$-bounded. Since $G$ is a finite $p$-group, there exists a normal subgroup $L$ in $G$ which is contained in $N$ and has index $p$ in $N$. By the assumption on $N$, the derived group of $G / L$ is not cyclic. Thus by factoring out $L$ we may assume that $N$ has order $p$ and therefore $G^{\prime}$ is 2-generator. Now Theorem 2.5 tells us that every element of $G^{\prime}$ is a commutator. Hence, it follows from Lemma 2.3 that $G^{\prime}$ has $m$-bounded order. The proof is complete.

\section{Proofs of the main Results}

We are now ready to complete the proofs of the theorems stated in the introduction.

Proof of Theorem A. By Lemma 2.2, it suffices to find a normal subgroup of $G$ inside $G^{\prime}$ with the desired properties. Let $\mathcal{N}$ be the family of all open normal subgroups of $G$, and observe that $G \cong \lim _{N \in \mathcal{N}} G / N$. Consider an arbitrary $N \in \mathcal{N}$, and put $Q=G / N$. Let us write $\mathcal{M}(N)$ for the set of all subgroups $R$ of $Q^{\prime}$ which are normal in $Q$, of order at most $f(m)$, and satisfy the condition that $Q^{\prime} / R$ is cyclic. By Theorem 2.8. $\mathcal{M}(N)$ is not empty.

Given $L, N \in \mathcal{N}$ with $L \leq N$, the natural map $\pi_{L N}$ from $G / L$ to $G / N$ induces a map $\varphi_{L N}$ from $\mathcal{M}(L)$ to $\mathcal{M}(N)$. This way we get an inverse system $\left\{\mathcal{M}(N), \varphi_{L N}, \mathcal{N}\right\}$ of finite sets. By [10, Proposition 1.1.4], the corresponding inverse limit is not empty. If $\left(M_{N} / N\right)_{N \in \mathcal{N}}$ is an element of that inverse limit, then $\pi_{L N}\left(M_{L} / L\right)=M_{N} / N$ for all $L, N \in \mathcal{N}$ such that $L \leq N$. Hence we can form the inverse limit $\lim _{N \in \mathcal{N}} M_{N} / N$, which corresponds to a normal closed subgroup $M$ of $G$. Since $\left|M_{N} / N\right| \leq f(m)$ for every $N \in \mathcal{N}$, we also have $|M| \leq f(m)$. Finally, observe that

$$
(G / M)^{\prime}=G^{\prime} / M \cong \lim _{N \in \mathcal{N}} \frac{(G / N)^{\prime}}{M_{N} / N}
$$

is an inverse limit of cyclic subgroups, and so procyclic.

Proof of Theorem B. Let $G$ be a pro- $p$ group such that all commutators in $G$ are covered by $m$ procyclic subgroups. Choose an open normal subgroup $N$ of $G$. The quotient $Q=G / N$ is a finite $p$-group satisfying the hypotheses of Theorem 2.9. Therefore either $Q^{\prime}$ is cyclic or the order of $Q^{\prime}$ is at most some $m$-bounded number $k$. Suppose now that the derived group $G^{\prime}$ is not of order at most $k$. Then there exists an open normal subgroup $N$ in $G$ such that the order of the derived group $(G / N)^{\prime}$ is larger than $k$ and hence $(G / N)^{\prime}$ is cyclic. Then $(G / H)^{\prime}$ is 
cyclic for any open normal subgroup $H$ contained in $N$. It follows that $G^{\prime}$ is procyclic, as required.

Proof of Theorem C. By the main result of [5] mentioned in the introduction, we know that $G^{\prime}$ is either cyclic or finite. So it is sufficient to concentrate on the case where $G^{\prime}$ is finite. There exists a finitely generated subgroup of $G$ whose derived subgroup coincides with $G^{\prime}$, and consequently we may assume that $G$ is finitely generated. As $G^{\prime}$ is finite, the centralizer $C_{G}\left(G^{\prime}\right)$ has finite index in $G$ and so it is also finitely generated. Moreover, $C_{G}\left(G^{\prime}\right)$ is nilpotent of class at most 2 and thus it is residually finite (see [7]). We conclude that $G$ is residually finite as well. Since $G^{\prime}$ is finite, there exists a normal subgroup $N$ of $G$ of finite index in $G$ such that $G^{\prime} \cap N=1$. As $G^{\prime}$ is isomorphic to $G^{\prime} N / N=(G / N)^{\prime}$ and in the finite group $G / N$ the result holds by Theorem 2.8, the conclusion follows.

\section{REFERENCES}

[1] C. Acciarri, P. Shumyatsky, On profinite groups in which commutators are covered by finitely many subgroups, Math. Z. 274 (2013), 239-248.

[2] C. Acciarri, P. Shumyatsky, On finite groups in which coprime commutators are covered by few cyclic subgroups, J. Algebra 407 (2014), 358-371.

[3] G. Cutolo, C. Nicotera, Verbal sets and cyclic coverings, J. Algebra 324 (2010), 1616-1624.

[4] E. Detomi, M. Morigi, P. Shumyatsky, On countable coverings of word values in profinite groups, J. Pure Appl. Algebra, to appear.

[5] G.A. Fernández-Alcober, P. Shumyatsky, On groups in which commutators are covered by finitely many cyclic subgroups, J. Algebra 319 (2008), 4844-4851.

[6] R. Guralnick, Commutators and commutator subgroups, Adv. Math. 45 (1982), 319-330.

[7] K.A. Hirsch, On infinite soluble groups, III, Proc. London Math. Soc. (2) 49 (1946), 184-194.

[8] M. Hall, Jr., The Theory of Groups, The Macmillan Co., New York, 1959.

[9] B. Huppert, Endliche Gruppen, Springer-Verlag, Berlin, 1967.

[10] L. Ribes, P. Zalesskii, Profinite Groups, Springer-Verlag, Berlin, 2000.

[11] D.J.S. Robinson, Finiteness conditions and generalized soluble groups, Part 1, Springer-Verlag, New York-Berlin, 1972.

[12] J.S. Wilson, Profinite Groups, Clarendon Press, Oxford, 1998. 
Department of Mathematics, University of the Basque Country UPV/EHU, 48080 Bilbao, Spain

E-mail address: gustavo.fernandez@ehu.es

Dipartimento di Matematica, Università di Bologna, Piazza di Porta San Donato 5, 40126 Bologna, Italy

E-mail address: marta.morigi@unibo.it

Department of Mathematics, University of Brasilia, Brasilia-DF, 70910-900 BRAZIL

E-mail address: pavel@unb.br 